Trabajos originales 


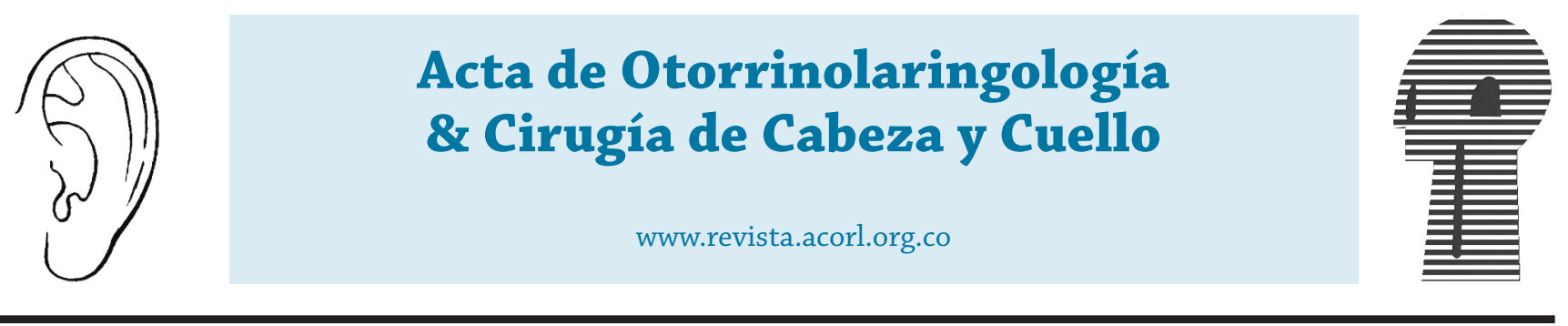

Trabajos originales

\title{
Manejo del tinnitus con estimulador de sonido con especificidad frecuencial
}

\section{Treatment of tinnitus with sound stimulator with frequency specificity}

\author{
José Eduardo Guzmán Durán*, Leonardo Elías Ordóñez Ordóñez*, Juanita María Beltrán Henríquez**, \\ Johanna Ximena Valderrama Penagos**, Néstor Ricardo González Marín ${ }^{* * *}$, Mónica Forero Llinás ${ }^{* * * *}$, \\ Alix Forero Acosta*****. \\ * $\quad$ Médico especialista en Otorrinolaringología, Otología y Neurootología. Especialista en docencia universitaria. Profesor \\ titular, Universidad Militar Nueva Granada. Servicio de Otorrinolaringología, Hospital Militar Central. Práctica privada. \\ ** Residente de Otorrinolaringología, Universidad Militar Nueva Granada. Hospital Militar Central. Bogotá, Colombia. \\ *** Médico especialista en Otorrinolaringología. Epidemiólogo. Profesor adjunto servicio de Otorrinolaringología, Hospital \\ Militar Central. \\ **** Fonoaudióloga, Escuela Colombiana de Rehabilitación, Universidad Iberoamericana. \\ ***** Fonoaudióloga. Audiología Mónica Forero Llinás. Bogotá, Colombia
}

Forma de citar: Guzmán JE, Ordonez-Ordonez LE, Beltran-Henriquez J, Valderrama-Penagos JX, Gonzalez-Marin NR, Forero M, Forero A. Manejo del tinnitus con estimulador de sonido con especificidad frecuencial. Acta otorrinolaringol. cir. cabeza cuello.2021;49(3): 184-188. DOI.10.37076/acorl.v49i3.607

\section{INFORMACIÓN DEL ARTÍCULO}

Historia del artículo:

Recibido: 16 de marzo de 2021

Evaluado: 20 de julio de 2021

Aceptado: 10 de agosto de 2021

Palabras clave (DeCS):

Tinnitus, cóclea, calidad de vida, hipoacusia.

\section{RESUMEN}

Introducción: el tinnitus tiene efectos deletéreos sobre la calidad de vida de un paciente. Cuando la lesión está a nivel coclear, se puede usar acondicionamiento acústico para su tratamiento. Objetivo: determinar el cambio en la percepción del tinnitus antes y después de la intervención terapéutica. Metodología: se planteó un estudio de serie de casos. Pacientes con tinnitus no pulsátil de moderado a catastrófico tratados con estimulador REVE $134^{\mathrm{TM}}$. Se incluyeron pacientes que no mejoraron luego de 3 meses con tratamiento médico. Se les practicó microaudiometría (67 frecuencias) para definir la región coclear afectada. Se excluyeron pacientes con umbrales audiométricos $>60 \mathrm{~dB}$, aquellos con lesiones retrococleares y quienes no desearon participar. Las variables de desenlace fueron Tinnitus Handicap Inventory (THI), escala visual análoga (EVA) y Tinnitus Reaction Questionnaire (TQR), que se midieron pretratamiento y a los 3 y 6 meses postratamiento. Resultados: se incluyeron 11 pacientes (hombres $=5$, mujeres $=6$ ). En 5 casos el tinnitus fue bilateral y

\section{Correspondencia:}

Leonardo Elías Ordóñez Ordóñez

Email: kokhlias@gmail.com

Dirección: Hospital Militar Central. Transversal 3 \#49-00, Bogotá, Colombia

Teléfono: 3113359835 
en 6, unilateral. Los valores pretratamiento fueron THI $=61,4 \pm 27,4, \mathrm{EVA}=6,9 \pm$ 2,7 y TQR $=43,2 \pm 31,9$ (Kolmogorov-Smirnov, $\mathrm{p}>0,05$ ). Hubo mejoría estadísticamente significativa con el tratamiento, THI $(3$ meses $=30,6 \pm 21,1 ; 6$ meses $=$ $19 \pm 19,2)$, EVA ( 3 meses $=5,6 \pm 2,3 ; 6$ meses $=3,5 \pm 2,0)$, TQR $(3$ meses $=25,6 \pm$ $20,0 ; 6$ meses $=14,3 \pm 19,9) ;$ ANOVA de medidas repetidas $(\mathrm{p}=0,007, \mathrm{p}=0,027, \mathrm{p}$ $=0,037$; respectivamente). Conclusión: el tratamiento con REVE $134^{\mathrm{TM}}$ fue efectivo en pacientes con tinnitus no pulsátil de moderado a catastrófico.

\section{ABSTRACT}

Key words (MeSH):

Tinnitus; Cochlea, Quality of Life, Hearing Loss.
Introduction: tinnitus can affect the quality of life of a patient. Acoustic stimulation can be used as treatment when the cause of tinnitus is located in the cochlea. Objective: To determine changes in tinnitus perception before and after therapeutic intervention. Methodology: We performed a case series study. Patients with nonpulsatile tinnitus, with no improvement with medical therapy, and moderate to catastrophic grade were treated with the REVE $134^{\mathrm{TM}}$ system. A microaudiometry (67 frequencies) was performed to determine the cochlear regions affected. Patients with auditory thresholds $>60 \mathrm{~dB}$, retrocochlear pathologies and who did not want to participate in the study were excluded. The variables studied were Tinnitus Handicap Inventory (THI), Visual Analog Scale (VAS) and Tinnitus Reaction Questionnaire (TQR), that were measured before, three and six months after treatment. Results: 11 patients (male: 5 , women: 6 ) were included. In 5 of them, tinnitus was bilateral and in 6, unilateral. Pretreatment values were: THI $=61.4 \pm 27.4$, VAS $=6.9 \pm 2.7$ and TQR $=43.2 \pm 31.9$ (Kolmogorov-Smirnov, $\mathrm{p}>0.05)$. We found improvement in tinnitus perception with the therapy, and this values had statistical significance (THI: 3rd month $=30.6 \pm 21.1 ; 6$ th month $=19 \pm 19.2)$, VAS $(3$ rd month $=5.6 \pm$ 2.3 ; 6th month $=3.5 \pm 2.0$ ), TQR (3rd month $=25.6 \pm 20.0$; 6th month $=14.3 \pm$ 19.9); repetitive measures of ANOVA ( $p=0.007, p=0.027, p=0.037$; respectively). Conclusion: Treatment with REVE $134^{\mathrm{TM}}$ was effective in patients with moderate to catastrophic tinnitus.

\section{Introducción}

Derivado del verbo latín tinnire (hacer sonar), el término tinnitus describe la percepción consciente de una sensación auditiva en la ausencia de un estímulo externo correspondiente. El tinnitus afecta entre un 10\%-20\% de la población general $(1,2)$ y para 12 millones de personas es lo suficientemente grave como para interferir con sus actividades diarias. Se conoce la relación entre la hipoacusia neurosensorial y el riesgo de desarrollo de tinnitus; sin embargo, el tinnitus puede estar presente independientemente del grado de pérdida auditiva (2).

El daño coclear de cualquier tipo, en caso de ser lo suficientemente intenso y extenso, conlleva a una reorganización del mapa cortical tonotópico. Esto quiere decir que las neuronas con una frecuencia característica dentro de la banda de frecuencia de la hipoacusia adquieren frecuencias características que corresponden a las frecuencias en los límites de la hipoacusia, debido a un aumento en la sensibilidad por las regiones vecinas, por lo que esta frecuencia, en el límite de la hipoacusia, se convierte en una frecuencia sobrerrepresentada a nivel central (más neuronas de lo normal son sensibles a esta frecuencia) (3). Se describe que esta reorganización central es la causante del tinnitus (4).
El tinnitus usualmente acompaña las hipoacusias neurosensoriales de frecuencias agudas y se ha considerado consecuencia de los cambios a nivel nervioso central consecutivos a una hipoacusia, y que a la larga estos cambios centrales pueden estar asociados con patrones aberrantes de descargas espontáneas interpretadas como sonidos reales (3, 5). Entonces, se describe que la presencia de estos patrones de descarga aberrante causan una disminución en el impulso excitatorio, que en consecuencia genera una disminución en la inhibición central. Esta disminución en la inhibición central puede inducir un desenmascaramiento de conexiones excitatorias latentes llevando a la producción de nuevas respuestas. La reorganización del mapa tonotópico posterior a una hipoacusia es la consecuencia de estos fenómenos $(6,7)$.

La terapia de acondicionamiento acústica se basa en que la pérdida auditiva lleva a cambios compensatorios a nivel central para mantener la actividad neural, y el tinnitus se genera como una consecuencia a estos cambios. Se han hecho hipótesis en cuanto a que el acondicionamiento acústico puede revertir esos cambios mal adaptativos por medio en un aumento en la estimulación neural. Para esto se ha descrito el uso de estímulos acústicos de banda ancha, amplificación del lenguaje y de sonidos ambientales por medio de audífonos para amplificar o proveer sonidos o música. El acondiciona- 
miento acústico se puede generar a niveles suficientes para hacer el tinnitus inaudible (enmascarar el tinnitus) o a niveles más bajos en los que aún se puede percibir el tinnitus (8).

Se ha desarrollado tecnología fundamentada en el acondicionamiento acústico, la cual requiere la realización de una microaudiometría con el fin de detectar las deflexiones negativas y definir el intervalo de frecuencias en las que se generará el estímulo acústico. El software Tinnitusless evalúa las características espectrales subjetivas de percepción (lateralidad, intensidad y frecuencia del tinnitus reportadas por el individuo) y usa la ilustración en medio digital de la cóclea con los diversos tonos (tono puro, tono de banda estrecha y tono mixto) que se generan en el rango de frecuencias específicas de 250 a $12000 \mathrm{~Hz}$, con el rango de intensidad de 0 a 84 decibelios relativos (dBr). El generador de estímulos REVE 134 es la abreviación de Restauración y Elevación de la Viscoelasticidad y Electromotilidad. Es un software capaz de generar estímulos entre $250-12000 \mathrm{~Hz}$ a una intensidad máxima de 84 decibelios (dB). Su funcionamiento está justificado en la búsqueda de las áreas específicas en la cóclea, basados en las frecuencias más afectadas en la microaudiometría, para generar una estimulación infraumbral dirigida hacia las células ciliadas externas de esta zona. Mediante la estimulación específica, se busca tener los efectos de restauración y elevación de la función de las células ciliadas externas, al evitar o modificar la reorganización de los mapas tonotópicos (9).

El objetivo de este estudio es describir los cambios en la calidad de vida en individuos con tinnitus posterior al uso del sistema REVE 134.

\section{Materiales y métodos}

Se realizó una serie de casos en la que se incluyeron 11 pacientes que consultaron a la práctica clínica de los autores entre noviembre de 2018 y junio de 2019. El protocolo fue aprobado previa realización de un comité interno por el grupo de investigación del servicio de otorrinolaringología.

Entre los criterios de inclusión se tuvo en cuenta presencia de tinnitus no pulsátil de muy leve a catastrófico, con hipoacusia neurosensorial entre 40-70 dB en frecuencias entre 250 y $12000 \mathrm{~Hz}$ que no mejoraron luego de tres meses con tratamiento médico.

Se les practicó una microaudiometría para definir la región coclear afectada y se excluyeron los pacientes que no cumplían con los criterios de inclusión. Posteriormente, se utilizó el software Tinnitusless para generar el mapa digital coclear y generar un estímulo individualizado. La estimulación se llevó a cabo por medio de 12 fases de acondicionamiento en períodos de 15 días calendario cada una, en las cuales se hizo uso de un estímulo dos veces al día en periodos de 40 minutos. Con el fin de evaluar el tinnitus y medir el impacto psicológico y la incapacidad que este le produce al individuo, se utilizó el instrumento propuesto por Newman y Jacobson en 1996 conocido como el Tinnitus
Handicap Inventory (THI) (10) al iniciar el tratamiento y a los 3 y 6 meses. Adicionalmente, se usaron los instrumentos de Tinnitus Reaction Questionnaire (TRQ) y la escala visual análoga (EVA).

Para el análisis se utilizó SPSS. Se realizó el análisis descriptivo de las variables, calculando la frecuencia relativa y absoluta de cada uno de los datos. Se utilizaron como variables de desenlace el THI, TQR y EVA. A estos datos se les calculó el mínimo, máximo, media y desviación estándar (DE). Además, se realizó prueba de Kolmogorov-Smirnov para verificar la distribución de las variables de desenlace. Se planteó el cálculo del análisis de varianza (ANOVA) para las variables de desenlace.

\section{Resultados}

Se realizó el estudio con 11 pacientes de los cuales 6 fueron mujeres $(54,5 \%)$ y 5 fueron hombres $(45,5 \%)$. El $63,6 \%$ de los pacientes no tenía una lateralidad específica del tinnitus y el 72,7 \% de los evaluados presentó acúfenos de instauración súbita. El 54,5 \% de la muestra evaluada no presentó ninguna otra comorbilidad y el 72,7 \% negó el consumo de medicamentos al momento del inicio del estudio. Además, 5 de los 11 pacientes incluidos tenían como profesión el hogar y solo uno tenía factor de riesgo ocupacional para hipoacusia por exposición a ruido crónica. El 63,3\% de los pacientes refería tinnitus fluctuante y el 54,5\% relacionaba el aumento del volumen del tinnitus a la exposición a ruido y estrés. El $81,8 \%$ de los pacientes refirió mayor frecuencia de tinnitus durante la noche y, de los 7 pacientes que presentaron tinnitus bilateral, 6 comentaron diferencias en los acúfenos entre ambos oídos. En cuanto a la caracterización psicoacústica del tinnitus, no hubo un patrón dominante. El $100 \%$ de los pacientes refirió la alteración en la calidad de vida con el tinnitus.

El THI pretratamiento (mínimo 18, máximo 98) presentó una media de $61,4 \pm 27,4$; la EVA (mínimo 3, máximo 10) presentó una media de $6,91 \pm 2,7$ y TQR (mínimo 7 , máximo 97) con promedio de 43,18 $\pm 31,9$. Se calculó $Z$ de Kolmogorov-Smirnov en el que se obtuvo una distribución normal para todas las variables $(\mathrm{p}>0,05)$. A los 3 meses del tratamiento, el THI tenía una media de 30,63 $\pm 21,15$ y $19 \pm$ 19,17 y a los 9 meses, de 19 (Tabla 1).

\begin{tabular}{|c|c|c|c|}
\hline \multicolumn{2}{|c|}{ Tabla 1. Media de THI con intervalos de confianza. } \\
\hline \multirow{2}{*}{ THI } & \multirow{2}{*}{ Media } & \multicolumn{2}{|c|}{ IC $95 \%$} \\
\cline { 3 - 4 } & & Límite inferior & Límite superior \\
\hline Inicial & 61,364 & 42,960 & 79,767 \\
\hline 3 meses & 30,636 & 16,429 & 44,844 \\
\hline 6 meses & 19,000 & 6,123 & 31,877 \\
\hline
\end{tabular}

IC: intervalo de confianza.

En lo que respecta a la EVA, la media a los tres meses fue de 5,55 $\pm 2,3$ y a los 6 meses fue de 3,45 $\pm 1,96$ (Tabla 2). 
Tabla 2. Media de la EVA con IC

\begin{tabular}{|c|c|c|c|}
\multirow{2}{*}{ EVA } & \multirow{2}{*}{ Media } & \multicolumn{2}{|c|}{ IC 95 \% } \\
\cline { 3 - 4 } & & Límite inferior & Límite superior \\
\hline Inicial & 6,909 & 5,120 & 8,698 \\
\hline 3 meses & 5,545 & 4,003 & 7,088 \\
\hline 6 meses & 3,455 & 2,132 & 4,777 \\
\hline
\end{tabular}

En cuanto al TQR, a los 3 meses de tratamiento la media fue de 25,64 $\pm 19,95$ y a los 6 meses fue de 14,27 $\pm 19,89$ (Tabla 3). El cálculo de ANOVA para el THI mostró una p de 0,007, el cálculo de ANOVA para EVA obtuvo una $\mathrm{p}=0,027$ $\mathrm{y}$, finalmente, el cálculo de ANOVA para TQR obtuvo una $\mathrm{p}$ $=0,037$.

\section{Tabla 3. Media de la TQR con IC}

\begin{tabular}{|c|c|c|c|}
\multirow{2}{*}{ TQR } & \multirow{2}{*}{ Media } & \multicolumn{2}{|c|}{ IC 95 \% } \\
\cline { 3 - 4 } & & Límite inferior & Límite superior \\
\hline Inicial & 43,182 & 21,749 & 64,614 \\
\hline 3 meses & 25,636 & 12,233 & 39,040 \\
\hline 6 meses & 14,273 & 0,907 & 27,638 \\
\hline
\end{tabular}

\section{Discusión}

El tinnitus puede afectar la vida diaria en múltiples aspectos como la generación de insomnio, dificultad para la concentración o para escuchar una conversación, trastornos de ansiedad y depresión, por lo que se ha asociado con una disminución en la calidad de vida y afectación psicosocial $(9,11)$. A pesar de que existen diferentes tipos de tratamiento para pacientes con tinnitus, como el uso de estímulos supraumbrales para enmascarar el tinnitus, medicamentos para tratar la ansiedad o el insomnio, terapias de relajación, terapia cognitiva comportamental, entre otros, no existe tratamiento que permita tratar la causa de la generación del tinnitus (12-14).

Entre las variantes del método de estimulación acústica se encuentra la estimulación acústica dirigida o acondicionamiento acústico, que busca prevenir la reorganización del mapa tonotópico y, de este modo, generar protección del sistema auditivo. Se basa en la estimulación de las frecuencias en la región correspondiente a la hipoacusia con el fin de compensar la disminución de la cantidad de descargas del nervio auditivo en estas zonas y, por tanto, prevenir la cascada de cambios a nivel central que normalmente produciría la reorganización del mapa cortical tonotópico (15). Estudios en ratones han demostrado que una disminución en las aferencias de la cóclea subyace a la degeneración del núcleo coclear anteroventral (NCAV) y, por tanto, la restauración o intensificación de estas aferencias tendría un efecto de mejoría en cuanto a la hipoacusia, degeneración coclear y cambios degenerativos en el NCAV. Los ratones tratados por medio de entornos acústicamente aumentados en altas frecuencias (centradas en $2000 \mathrm{~Hz}$ ) mostraron menor pérdida neuronal y menor pérdida de volumen de tejido en la porción dorsal del NCAV en comparación con los controles. Adicionalmente, la exposición prolongada a un entorno acústicamente aumentado puede retrasar y disminuir los cambios relacionados con la edad, siempre y cuando el entorno acústico sea provisto previo al desarrollo de una hipoacusia grave (16).

El acondicionamiento acústico puede realizarse por medio de métodos tempranos o tardíos. Por una parte, el método temprano consiste en la exposición previa a niveles moderados de sonido, los cuales reducen la hipoacusia inducida por exposición al ruido. Por otra parte, el método tardío involucra el uso de un estímulo acústico posterior a la exposición a un ruido intenso que ha mostrado efectos protectores contra el trauma acústico, lo que previene la reorganización del mapa tonotópico cortical $(8,15)$.

Una de las alternativas actuales disponibles para el manejo del tinnitus es el sistema REVE 134. A pesar de ser una terapia recientemente desarrollada, su fundamento teórico hace pensar que podría ser una herramienta útil para el manejo del tinnitus. En este estudio se evidenció una disminución significativa de los cuestionarios de tinnitus con el inicio de tratamiento con REVE 134 y se encontraron diferencias hasta de un $69 \%$ (THI) en el cuestionario inicial frente al cuestionario realizado a los 6 meses. Los resultados obtenidos mostraron significación estadística con las pruebas de ANOVA.

El tratamiento con REVE 134 en el estudio resultó ser efectivo en pacientes con tinnitus no pulsátil de grado moderado a catastrófico; sin embargo, los resultados obtenidos no son comparables en la literatura debido a la falta de publicación de artículos y a la abundancia de literatura gris.

\section{Conclusiones}

El tratamiento con REVE 134 muestra eficacia en el tratamiento del tinnitus y genera mejoría en la calidad de vida. Sin embargo, es necesaria la realización de más investigaciones para objetivar los datos obtenidos en esta investigación.

Una de las limitaciones presentadas durante esta investigación es el número de la muestra. Consideramos que la realización de estudios posteriores podrá mostrar resultados similares a los encontrados confirmando la efectividad del tratamiento con estimulador REVE 134.

\section{Conflicto de intereses}

Los autores declaramos el apoyo de la casa comercial Ear Logic con sus productos AMA-PTA (microaudiometría), Tinnitusless (examen diagnóstico de tinnitus) y REVE 134 (tratamiento de sonido condicionado).

Contribución de los autores en las etapas y concepción del artículo.

Todos los autores contribuyeron en el diseño del estudio, la selección de los candidatos, la recolección e interpretación de los datos y la redacción del presente estudio. 


\section{Aspectos éticos}

De acuerdo con los principios éticos de la declaración de Helsinki y la Resolución n. 008430 de 1993 del Ministerio de Salud y en la Ley 84 de 1989, se considera un estudio con riesgo mínimo.

\section{REFERENCIAS}

1. Longenecker RJ, Galazyuk AV. Methodological optimization of tinnitus assessment using prepulse inhibition of the acoustic startle reflex. Brain Res. 2012;1485:54-62. doi: 10.1016/j. brainres.2012.02.067

2. Shargorodsky J, Curhan SG, Curhan GC, Eavey R. Change in prevalence of hearing loss in US adolescents. JAMA. 2010;304(7):772-8. doi: 10.1001/jama.2010.1124

3. Noreña AJ, Eggermont JJ. Enriched acoustic environment after noise trauma reduces hearing loss and prevents cortical map reorganization. J Neurosci. 2005;25(3):699-705. doi: 10.1523/ JNEUROSCI.2226-04.2005

4. Noreña AJ, Eggermont JJ. Changes in spontaneous neural activity immediately after an acoustic trauma: implications for neural correlates of tinnitus. Hear Res. 2003;183(1-2):137-53. doi: 10.1016/s0378-5955(03)00225-9

5. Calford MB. Dynamic representational plasticity in sensory cortex. Neuroscience. 2002;111(4):709-38. doi: 10.1016/ s0306-4522(02)00022-2

6. Noreña AJ, Tomita M, Eggermont JJ. Neural changes in cat auditory cortex after a transient pure-tone trauma. J Neurophysiol. 2003;90(4):2387-401. doi: 10.1152/ jn.00139.2003

7. Norena A, Micheyl C, Chéry-Croze S, Collet L. Psychoacoustic characterization of the tinnitus spectrum: implications for the underlying mechanisms of tinnitus. Audiol Neurootol. 2002;7(6):358-69. doi: 10.1159/000066156
8. Willott JF. Effects of sex, gonadal hormones, and augmented acoustic environments on sensorineural hearing loss and the central auditory system: insights from research on C57BL/6J mice. Hear Res. 2009;252(1-2):89-99. doi: 10.1016/j. heares.2008.12.002

9. Kwak E, Kwak S, Song S, Kim S, Hong S. A new method for restoration of sensorineural hearing loss: a prospective clinical study. Seúl: Samsung Medical Center; 2012 [consultado el falta la fecha en que el autor consultó el enlace]. Disponible en: https://biosom.com.br/restoration_of_hearing_loss.pdf

10. Baguley DM, Andersson G. Factor analysis of the Tinnitus Handicap Inventory. Am J Audiol. 2003;12(1):31-4. doi: 10.1044/1059-0889(2003/007)

11. Nondahl DM, Cruickshanks KJ, Huang GH, Klein BE, Klein $\mathrm{R}$, Nieto FJ, et al. Tinnitus and its risk factors in the Beaver Dam offspring study. Int J Audiol. 2011;50(5):313-20. doi: 10.3109/14992027.2010.551220

12. Tyler RS, Baker LJ. Difficulties experienced by tinnitus sufferers. J Speech Hear Disord. 1983;48(2):150-4. doi: 10.1044/jshd. 4802.150

13. Tunkel DE, Bauer CA, Sun GH, Rosenfeld RM, Chandrasekhar SS, Cunningham ER Jr, et al. Clinical practice guideline: tinnitus. Otolaryngol Head Neck Surg. 2014;151(2 Suppl):S1-S40. doi: 10.1177/0194599814545325

14. Auerbach BD, Rodrigues PV, Salvi RJ. Central gain control in tinnitus and hyperacusis. Front Neurol. 2014;5:206. doi: 10.3389/fneur.2014.00206

15. Willott JF, Bosch JV, Shimizu T, Ding DL. Effects of exposing $\mathrm{DBA} / 2 \mathrm{~J}$ mice to a high-frequency augmented acoustic environment on the cochlea and anteroventral cochlear nucleus. Hear Res. 2006;216-217:138-45. doi: 10.1016/j. heares.2006.01.010

16. Willott JF, Turner JG, Sundin VS. Effects of exposure to an augmented acoustic environment on auditory function in mice: roles of hearing loss and age during treatment. Hear Res. 2000;142(1-2):79-88. doi: 10.1016/s0378-5955(00)00014-9 